\title{
Emerging trends and applications in ad hoc networks
}

\author{
Luigi Fratta ${ }^{1}$ Mario Gerla ${ }^{2} \cdot$ Keun-Woo Lim $^{3}$ \\ Published online: 18 August 2018 \\ (C) Institut Mines-Télécom and Springer Nature Switzerland AG 2018
}

After our successful hosting of the 16th Annual Mediterranean Ad Hoc Networking Workshop (Medhocnet 2017) in Budva, Montenegro, we are pleased to present some of the best works of the workshop in the special issue of the Annals of Telecommunications, "Emerging Trends and Applications in Ad Hoc Networks." The workshop, which was held in June 28, 2017-June 30, 2017, featured very interesting research in the field of mobile ad hoc networks (MANETs) that represents the future trend of MANET services and applications.

In contrast to the several decades of existing research, we are seeing an evolution in this field of MANET, mainly due to the development of exciting new hardware (UAVs, intelligent drones, smart vehicles, etc.) and software (embedded platforms). Even in the field of sensor networks, a major component of MANET, we witness the emergence of more service/ application-oriented research topics well-integrated with the industry and the commercial services of Internet-of-Things (IoT) such as smart grid, smart vehicles, and smart home. Indeed, it is truly an exciting new trend that we are facing, characterized by many new challenges.

The main objective of this special issue was to capture these new trends in MANETs and publish high-quality research relevant to it. The papers offer a very wide range of research, not only on theoretical aspects but also on experimental research implementations on actual mobile devices and testbeds. In total, 18 submissions were received through the open Call for Papers. At least two thorough reviews were given for each paper, thanks to the contributions of the many reviewers with a diverse range of expertise not only in MANET but also in security, communications, localization, quality-of-service, and more. After a careful reviewing process, we were able to select

Keun-Woo Lim

keunwoo.lim@telecom-paristech.fr

Politecnico di Milano, Milan, Italy

University of California, Los Angeles, Los Angeles, CA, USA

3 Telecom Paristech, Paris, France seven high-quality research papers. It was interesting to note that the accepted papers can be grouped and mapped to specific MANET emerging topics. We exploit this grouping to summarize and present these works.

First of all, the majority of the accepted papers had their topics focused on mobile opportunistic networking scenarios with delay-tolerant networking characteristics.

In recent years, one of the most active areas in MANETs has been delay-tolerant networking (DTN). A DTN may become temporarily disconnected into subnetworks because of node movements, obstacles in the environment, or inadequate transmission range. Examples include vehicular networks, tactical networks, and pedestrian smartphone networks. If a node cannot reach the destination of the data packet it needs to forward, it stores the packet and, whenever it meets another moving node, decides based on utility functions if the new neighbor is a promising relay to reach destination and if it behooves to forward the packet to it. Obviously, delivery times cannot be guaranteed, delays must be tolerated, hence the name "DTN." Each node, as it moves, constructs a graph with history of reachable neighbors (with one, two of more hops). The graph is used to compute utility. The two DTN papers in the special issue are based on this concept, but introduce innovations of their own.

The paper "Time-Constrained Anycast Routing under Short Contact Duration in Delay Tolerant Networks" by Tuan Lee and Mario Gerla adds the novelty of single copy ANYCAST routing among eligible destinations, subject to time constraints. E.g., in an urban emergency network, an ambulance must be called. The proposed scheme reaches the nearest ambulance within tolerable delay. It shows major improvements in delivery rate, delay, and traffic overhead with respect to the state of the art.

The paper "Context-Adaptive Forwarding in Mobile Opportunistic Networks" by Radu Ciobanu, Daniel Gutierrez, Ciprian Dobre, and Sergio Toral introduces the new concept of "context" in the utility function definition. That is, the decision to forward the packet, or which packet to forward, is not based on a time-invariant utility function, rather on a function that adapts to 
the context in which the node finds itself, for example, disconnected component size. The authors claim that context awareness significantly enhances hit ratio, latency, and transmission costs.

While having somewhat similar characteristics to mobile opportunistic networking, Eirini Kalogeiton, Thomas Kolonko, and Torsten Braun place emphasis on vehicular ad hoc networks (VANETs) in their work "A topology-oblivious routing protocol for NDN-VANETs." To cope with the intermittent connectivity induced from high-speed vehicles, the authors design a multi-hop routing protocol which leverages multi-path/multi-channel communication to enable connectivity between nodes. Furthermore, they use Named Data Networking (NDN) technology to enable vehicles to search and route to specific content of interest, instead of connecting to other nodes following the traditional IP/MAC addressing system.

Different from the aforementioned works, in " $\mu$ DTNSec: A security layer with lightweight certificates for DisruptionTolerant Networks on microcontrollers," the authors Dominik Schürmann, Georg von Zengen, Marvin Priedigkeit, Sebastian Willenborg, and Lars Wolf focus on the security aspects of the DTN environment. Their main goal is to develop a lightweight key infrastructure for sensor nodes, implemented and tested in an actual sensor testbed based on IEEE 802.15.4 and Contiki operating system. This allows signature verification between nodes in an end-to-end connection manner. Furthermore, for the ease of managing sets of sensors, the authors also design a certificate-based key sharing method which can conveniently update sensors in case of node addition.

The next two papers address an interesting common topic in the field of flying ad hoc networks (FANETs), enabled through the development of drones and UAVs. Due to the high mobility of such flying devices, maintaining a satisfactory quality of data streaming is a challenging issue, not to mention the energy efficiency problem of battery-powered devices. Jośe Filho, Danileno Rośario, Denis Rośario, Aldri Santos, and Mário Gerla, in their work "Satisfactory Video Dissemination on FANETs Based on An Enhanced UAV Relay Placement Service," focus on the point that the mobility of UAVs often induces communication and networking problems, especially if the UAVs must be organized in a multi-hop network. These problems become even more critical when video streaming is considered, as the latter requires a high level of quality of experience (QOE) for accurate information reception. To solve this problem, this work proposes MobiFANET, a strategy that utilizes the deployment of relay drones in specific locations of the network, to enable geographic routing protocols to satisfy the required quality levels of video transmission.

In the same theme, Woo-Sung Jung, Jinhyuk Yim, and Young-Bae Ko, in their work "Adaptive Offloading with MPTCP for Unmanned Aerial Vehicle Surveillance System," also consider FANETs for video surveillance, but instead address the flight time and energy efficiency problems of UAVs. For UAVs, the operation time is very sensitive to computational and communication overhead. Specifically, UAVs can be performance-limited in computing resources, which makes it desirable to offload specific computing tasks to a more powerful gateway device. This work shows how offloading of video data processing can reduce time of task completion, while in fact it also saves the energy consumed by UAVs per task, significantly improving task completion rate and operation time efficiency. Furthermore, usage of Multipath TCP (MPTCP) provides a more reliable wireless transmission of tasks, which is necessary in a dynamic FANET network.

Shisupal Kumar, Nidhi Lal, and Vijay Chaurasiya focus on the more traditional sensor network architecture in their work "A Forwarding Strategy based on ANFIS in Internet-of-things oriented Wireless Sensor Network (WSN) using a Novel Fuzzy-Based Cluster Head Protocol." However, they apply some novel twists compared to existing work. Namely, the authors use data fusion (aggregation) features for enhanced energy efficiency and emphasize the usage of clustered networks. In their work, they approach the cluster head selection problem in WSN with considerations on energy, density, throughput, and distance information. Furthermore, to cope with the unreliable transmission environment, the authors apply neural network-based learning technique grounded on fuzzy logic principles to select multi-hop forwarding paths and allow more efficient data transmission in large-scale sensor networks.

To conclude, the Guest Editors would like to thank the reviewers for their contributions and insights which helped shape this special issue in the Annals of Telecommunications. We also thank the editorial office of ANTE for the efficient management and feedback in the overall organization of this special issue. Most importantly, we express our gratitude to the authors for contributing their interesting, original research. We hope that this special issue will offer new insights and perspectives to future researchers that will follow and reference these papers in their work. 